\title{
Variations in xanthophyll composition in etiolated seedlings of Arabidopsis thaliana correlate with protochlorophyllide accumulation*
}

\author{
Beata Myśliwa-Kurdziel, Małgorzata Jemioła-Rzemińska, Elżbieta Turek, Kazimierz Strzałka \\ and Przemysław Malec
}

Department of Plant Physiology and Biochemistry, Faculty of Biochemistry, Biophysics and Biotechnology, Jagiellonian University, Kraków, Poland

\begin{abstract}
Protochlorophyllide (Pchlide) accumulation and xantophyll composition were studied in 5-day old etiolated seedlings of three ecotypes of Arabidopsis thaliana: Columbia (Col-0), Landsberg erecta (Ler) and Wassiliewska (Ws). The total Pchlide level as measured by fluorescence spectroscopy varied significantly between ecotypes. A rapid HPLC method revealed quantitative differences in carotenoid composition. It was found that in the Ler ecotype any enhanced accumulation of Pchlide correlates with an increased level of lutein, suggesting the role of enzymes involved in lutein synthesis in cross-regulation between chlorophyll and carotenoid biosynthetic pathways. The function of the dark-accumulated carotenoid pool in seedling de-etiolation is discussed.
\end{abstract}

Key words: carotenoids, de-etiolation, HPLC, protochlorophyllide

Received: 19 October, 2011; accepted: 01 March, 2012;

available on-line: 17 March, 2012

\section{INTRODUCTION}

Angiosperms require light for their morphogenesis. While growing in the dark, their seedlings become etiolated. With long hypocotyls and small yellowish cotyledons (Fig. 1A), they develop etioplasts containing paracrystalline prolamellar bodies, instead of chloroplasts. Prolamellar bodies are lipid structures containing compounds important for the protection of developing seedlings from oxidative stress induced by illumina-

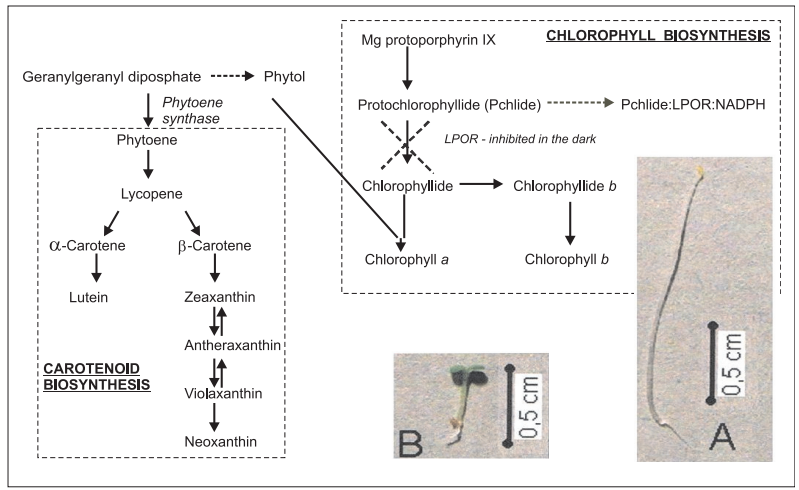

Figure 1. A simplified scheme of carotenoid and chlorophyll biosyntheses.

LPOR- light-dependent protochlorophyllide oxidoreductase. Photo: etiolated (A) and green (B) 5-day-old Arabidopsis seedling. tion (reviewed by Solymosi \& Schoefs, 2010). Chlorophyll biosynthesis is stopped in darkness at the stage of the formation of protochlorophyllide (Pchlide; Fig. 1), and Pchlide is accumulated in etioplasts (reviewed by: Schoefs \& Franck, 2003; Schoefs, 2005; Belyaeva \& Litvin, 2007; Myśliwa-Kurdziel \& Strzałka, 2010). The first exposure of emerging seedlings to light evokes a switch to photoautotrophy. This process (de-etiolation, photomorphogenesis) is controlled by an interactive network of multiple photoreceptors (predominantly phytochromes and cryptochromes) and their downstream signaling elements (reviewed by: Casal, 2006). De-etiolation is accompanied with the light-regulated differential expression of many nuclear-and plastid-encoded genes and induces profound changes in plant morphology (Fig. 1A, B) and physiology (reviewed by: von Arnim \& Deng, 1996). The light-triggered reduction of Pchlide to chlorophyllide is a prerequisite for chlorophyll biosynthesis and, in consequence, the formation of a fully functional photosynthetic apparatus.

Carotenoids are photoprotective and antioxidant pigments synthesized in all photosynthetic organisms. Etiolated seedlings accumulate xanthophylls, mainly lutein and violaxanthin, formed in separated branches of the carotenoid biosynthesis pathway (Fig. 1; see: Cazzonelli \& Pogson, 2010 for a review). Although the accumulation of all-trans-xanthophylls has been found to be essential for prolamellar body formation and thus for the efficiency of Pchlide photoreduction (Park et al., 2002; Cuttris et al., 2007), the detailed physiological function of the carotenoid pool during de-etiolation remains unknown.

Previous studies have revealed that the biosynthesis of carotenoids is regulated by light via the phytochromecontrolled level of phytoene synthase, the first enzyme of the pathway (Welsch et al., 2000; Fig. 1). Phytoene synthase has been postulated recently as a key target for light and hormonal signaling networks in plants (see: Rodriguez-Villalón et al., 2009 for a review). Moreover, $\xi$-carotene desaturase has recently been shown to be involved both in chloroplast development and plastid-tonucleus retrograde signaling (Dong et al., 2007). Thus, it seems likely that at the early stages of seedling development the functioning of both carotenoid and chlorophyll

e-mail: przemyslaw.malec@uj.edu.pl

*Presented at the 16th International Symposium on Carotenoids, 17- 22 July, 2011, Kraków, Poland

Abbreviations: Col-0, Columbia; Ler, Landsberg erecta; LPOR, lightdependent protochlorophyllide oxidoreductase; Pchlide, protochlorophyllide; Ws, Wassiliewska. 
biosynthesis pathways is orchestrated at the level of cellular regulation (Meier et al., 2011).

Recently we have found that the etiolated Arabidopsis seedlings of photoreceptor mutant lines originating from several background ecotypes differ significantly in their ability to accumulate Pchlide (Myśliwa-Kurdziel et al., in press). In the present study using the etiolated seedlings of three ecotypes of Arabidopsis thaliana: Columbia (Col-0), Landsberg erecta (Ler) and Wassiliewska (Ws) we show that the level of Pchlide accumulation is accompanied by differences in xanthophyll composition, providing evidence that enzymes of the carotenoid biosynthesis pathway downstream of phytoene synthase and $\xi$-carotene desaturase may be involved in the regulation of greening capacity in angiosperms.

\section{MATERIALS AND METHODS}

Arabidopsis thaliana ecotypes. Columbia (Col-0), Landsberg erecta (Ler) and Wassiliewska (Ws) were obtained from the Arabidopsis Biological Resource Center, Ohio State University, Ohio, USA. The seedlings were grown for 5 days on sterile MS-agar plates at $22^{\circ} \mathrm{C}$ in complete darkness, as described by Malec et al. (2002). Approximately $100 \mathrm{mg}$ of cotyledons was homogenized in 5-7 $\mathrm{ml}$ of acetone with a pestle and mortar. The supernatant was collected and the extraction procedure was repeated three times. All the extracts were combined by vortexing, clarified by centrifugation $(10000 \times g$ for 5 min) and used directly for the estimation of Pchlide content.

The relative Pchlide content in etiolated seedlings was calculated from the fluorescence emission spectra of acetone extracts recorded at room temperature. The fluorescence spectra were measured with a Perkin-Elmer LS-50 spectrofluorometer for excitation at $440 \mathrm{~nm}$; the excitation and emission slits were 10 and 5, respectively. The extracts were diluted before measurement, so as to have a linear correlation between fluorescence intensity and pigment concentration, and the dilution was taken into account in calculations. The fluorescence intensity at $633 \mathrm{~nm}$ (i.e. at the maximum) as read from the spectrum per gram of the fresh weight of the plant tissue is taken as a measure of Pchlide accumulation.

For carotenoid analysis the acetone extract was evaporated to dryness with gaseous nitrogen. The film obtained was dissolved directly in the HPLC mobile phase (acetonitrile:methanol: $\mathrm{H}_{2} \mathrm{O}$ 72:8:1; v/v/v). The HPLC system consisting of a quaternary pump, a vacuum degasser, an autosampler and a diode array detector (Agilent 1200 series) was controlled by Agilent ChemStation software. An aliquot of $50 \mu \mathrm{L}$ sample was injected onto any Agilent Zorbax Eclipse Plus C18 (4.6×150 mm; 3.5 $\mu \mathrm{m}$ pore size) column, equipped with a guard column. The elution was performed in the isocratic mode with a flow rate of $0.7 \mathrm{~mL} \cdot \mathrm{min}^{-1}$. Carotenoids were identified on the basis of their absorption spectra. All experiments were repeated at least five times and the average relative content of each carotenoid identified was calculated as a percentage of the total area under the peaks on the chromatogram recorded at $460 \mathrm{~nm}$.

The statistical significance of the experimental data was evaluated by using a $t$-test at $p<0.05$.

\section{RESULTS AND DISCUSSION}

The accumulation of Pchlide varied significantly between the three Arabidopsis ecotypes (Col-0, Ler, Ws)

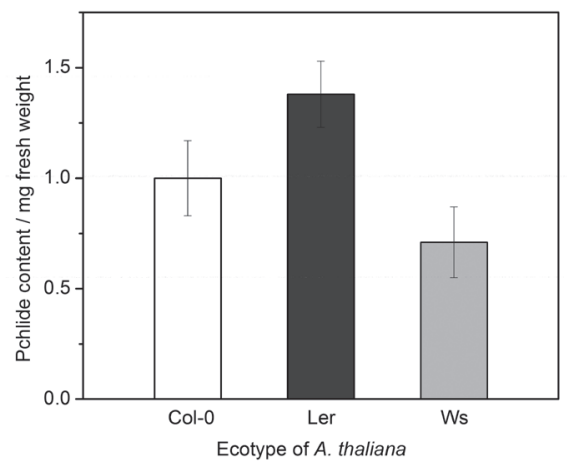

Figure 2. Relative protochlorophyllide content in different ecotypes of Arabidopsis (Col-0, Columbia; Ler, Landsberg erecta; Ws, Wassiliewska) as measured by fluorescence spectroscopy (see Materials and Methods for details).

grown for 5 days in the dark under controlled conditions. The relative Pchlide amount in Ler was the highest among the ecotypes, exceeding by ca. $30 \%$ the value observed for Col-0 and by ca. 50\% for Ws (Fig. 2).

A representative HPLC elution profile of pigment extract from the cotyledons of 5-day-old dark-grown $A$. thaliana seedlings is shown in Fig. 3A. The peaks are well resolved and the absorption spectra can be easily analyzed. Porphyrin derivatives were distinguished from carotenoids, and up to 13 carotenoid-type compounds were detected. The respective absorption spectra are shown in Fig. 3 (B-D). Eight carotenoid constituents were identified on the basis of the absorption spectra. The identification of the other compounds separated under our experimental conditions designated in the present paper by the numbers 5 and 10-13 needs further investigation. These results are in line with the earlier observations reported by Schoefs et al. (1995) for bean leaves. However, in the method used by these authors, some carotenoid peaks did not reach a separation level that would allow an efficient quantitative analysis of them. Taking advantage of the method described here the carotenoid composition can be accomplished rapidly and simultaneously in a single chromatographic run. It yields well-separated peaks and is suitable for the analysis of numerous samples.

The method was applied to the analysis of the carotenoid content of etiolated Arabidopsis seedlings. In each ecotype (Col-0, Ler, Ws) a similar carotenoid composition was observed, with trans-lutein and trans-violaxanthin being the most abundant xanthophylls (Fig. 3). However, there were remarkable quantitative differences between the ecotypes when it comes to the relative content of these two xantophylls. Particularly in Ler, the level of trans-lutein was ca. 12\% higher, whereas that of trans-violaxanthin was significantly lower (ca. 25\%) with respect to the results obtained for Col-0 and Ws, for which a similar level of trans-lutein and trans-violaxanthin abundance was found. Statistically significant differences between Ler and the two other ecotypes were also observed for the accumulation of lutein-5,6-epoxide and for two unidentified carotenoid-type compounds, shown as peaks No 5 and No 12 on Fig. 3.

The results presented in this communication show a significant variation in the accumulation of Pchlide in etiolated wild-type Arabidopsis seedlings of different ecotypes growing in the dark under controlled conditions. These differences may reach $30-50 \%$ between the ecotypes. Arabidopsis ecotypes represent stable lines which express natural genetic variation within a species 

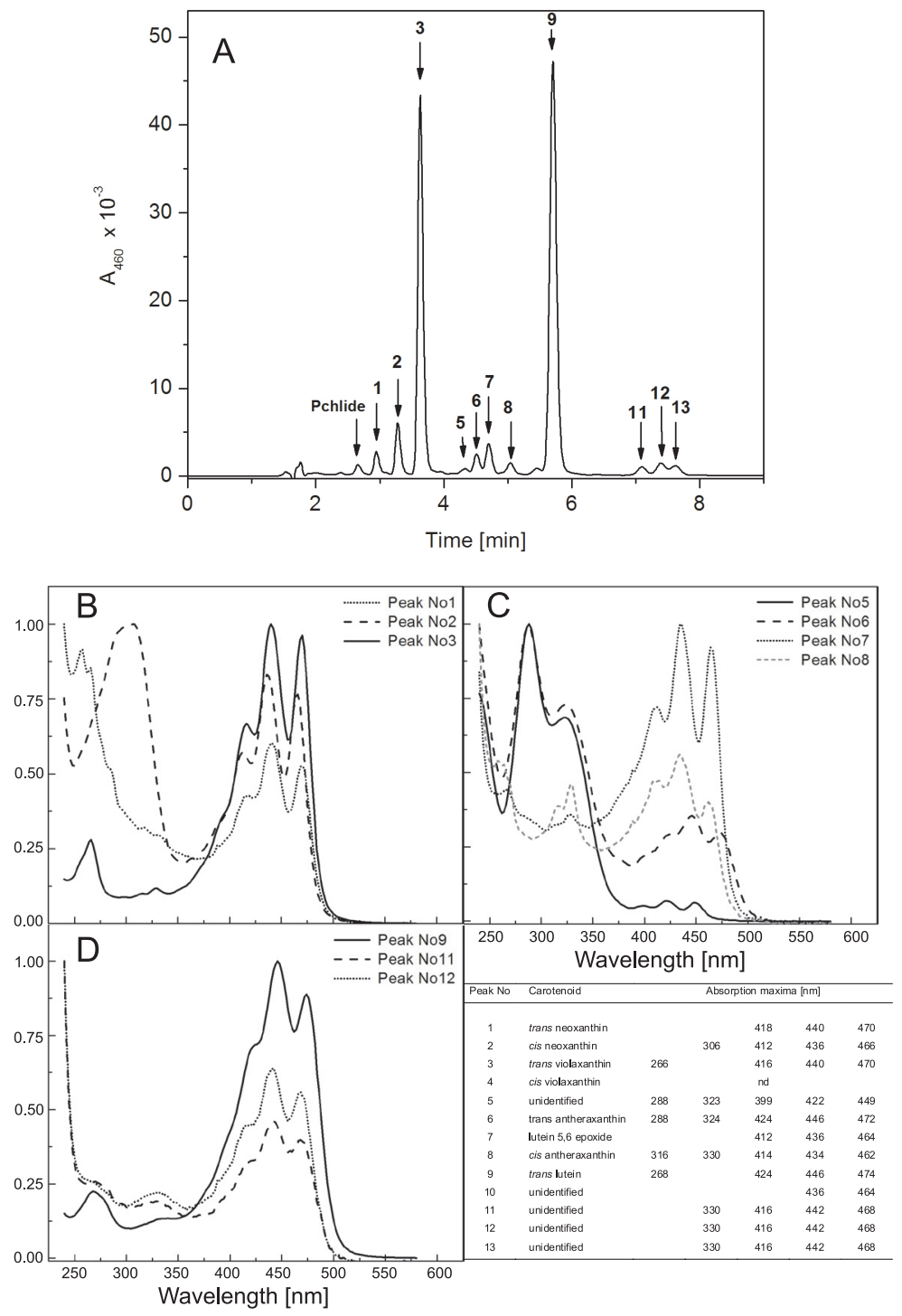

Figure 3. Reversed- phase HPLC of pigments extracted from 5-day-old A. thaliana (Col-0) seedlings (A). Chromatogram recorded at $460 \mathrm{~nm}$. Absorption spectra of detected carotenoids (B-D) as well as their absorption maxima (Table). Identification was based on the absorption spectrum and retention time.

(Koornneef \& Kendrick, 1994). It can be concluded, therefore, that the capacity for Pchlide biosynthesis and its subsequent accumulation in the dark may be dependent on the genetic background. In particular, the differences in phytochrome-controlled greening capacity as measured by chlorophyll accumulation, which were observed between ecotypes Col-0 and Ler have been shown to be the result of genetic polymorphisms in loci VLF1 and VLF2 (Yanovsky et al., 1997). The analysis of carotenoid composition clearly demonstrated that Ler seedlings, accumulating high amounts of Pchlide, expressed an increased level of lutein and a decreased level of violaxanthin. This result suggests an apparent upregulation of the branch of the carotenoid biosynthesis pathway initiated by lycopene $\varepsilon$-cyclisation accompanied by a downregulation of $\beta$-cyclisation branch. An increased accumulation of Pchlide has been recently uncovered in dark-grown barley seedlings treated with a herbicide which induces the inhibition of $\beta$-lycopene cyclase. The existence of mechanisms for enhanced tetrapyrolle accumulation stimulated by the accumulation of linear carotenoids were then proposed (La Rocca et al., 2007).

Lutein has been found to be essential for prolamellar body formation. A complete loss of this structure was observed in the cor mutant of Arabidopsis which had impaired lutein content (Cuttris et al., 2007). The formation of a prolamellar body has been found to correlate with the accumulation of Pchlide existing within Pchlide:LPOR:NADPH complexes in dark-grown Arabidopsis seedlings (Franck et al., 2000). It has also been reported that increased carotenoid accumulation in etiolated leaves causes a decrease in the efficiency of Pchlide photoreduction as well as influences the spectral properties of newly formed

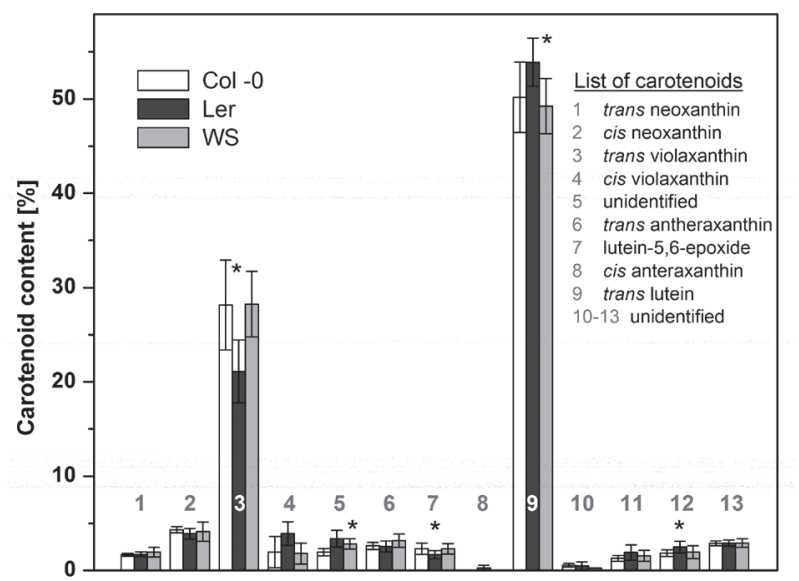

Figure 4. The relative carotenoid content determined in the seedlings of the investigated $A$. thaliana ecotypes expressed as percentage of the total area under all carotenoid peaks.

The presented values are means \pm standard deviation. An asterisk $\left(^{*}\right)$ indicates significant differences at $p<0.05$. chlorophyllide during seedling de-etiolation under light of low intensity (Yahubyan et al., 2001). Thus, the increased trans-lutein accumulation in Ler may affect both the recruitment of Pchlide molecules into complexes formed in the prolamellar body and their photoreduction during de-etiolation.

In conclusion, the results presented here show that the accumulation of carotenoids, especially trans-lutein and trans-violaxanthin, in etiolated seedlings of Arabidopsis differs between ecotypes. The enhanced accumulation of Pchlide correlates with an increased level of lutein, suggesting the role of enzymes involved in lutein synthesis in cross-regulation between chlorophyll and carotenoid biosynthetic pathways. The detailed mechanisms orchestrating these processes remain to be determined.

\section{Acknowledgements}

This work was supported by grant No N N303 498438 from The Polish Ministry of Science and Higher Education. The authors thank to Justyna Stępien and Paulina Chyl for a technical help in the preparation of 
samples for HPLC. The research was carried out with the equipment purchased thanks to the financial support of the European Regional Development Fund in the framework of the Polish Innovation Economy Operational Program (contract No. POIG.02.01.00-12-167/08, project Małopolska Center of Biotechnology).

\section{LITERATURE}

Belyaeva OB, Litvin FF (2007) Photoactive pigment-enzyme complexes of chlorophyll precursor in plant leaves. Biochemistry Moscow 72: 1458-1477.

Casal JJ (2006) The photoreceptor interaction network. In: Photomorphogenesis in Plants and Bacteria $3^{\text {rd }}$ ed. Schafer E, Nagy F, eds, pp 407-437. Springer, Netherlands.

Cazzonelli CI, Pogson BJ (2010) Source to sink: regulation of carotenoids biosynthesis in plants. Trends Plant Sci 15: 266-274.

Cuttris AJ, Chubb AC, Alawady A, Grimm B, Pogson BJ (2007) Regulation of lutein biosynthesis and prolamellar body formation in Arabidopsis. Funct Plant Biol 34: 663-672.

Dong H, Deng Y, Mu J, Lu Q, Wang Y, Xu Y, Chu C, Chong K, Lu C, Zuo J (2007) The Arabidopsis Spontaneous Cell Death1 gene, encoding a zeta-carotene desaturase essential for carotenoid biosynthesis, is involved in chloroplast development, photoprotection and retrograde signalling. Cell Res 17: 458-470.

Franck F, Sperling U, Frick G, Pochert B, van Cleve B, Apel K, Armstrong GA (2000) Regulation of etioplast pigment protein complexes, inner membrane architecture, and protochlorophyllide $a$ chemical heterogeneity by light-dependent NADPH:protochlorophyllide oxidoreductase A and B. Plant Physiol 124: 1678-1696.

Koornneef M, Kendrick RE (1994) Photomorphogenic mutants of higher plants. In: Photomorphogenesis in plants, Kendrick RE, Kronenberg GHM, eds, pp 601-628, Kluwer Academic Publishers, Dordrecht.

La Rocca N, Rascio N, Öster W, Rüdiger W (2007) Inhibition of lycopene cyclase results in accumulation of chlorophyll precursors. Planta 225: 1019-1029.

Malec P, Yahalom A, Chamovitz DA (2002) Identification of a lightregulated protein kinase activity from seedlings of Arabidopsis thaliana. Photochem Photobiol 75: 178-183.
Meier S, Tzafdia O, Vallabhaneni R, Gehring Ch, Wurtzel ET (2011) A transcriptional analysis of carotenoid, chlorophyll and plastidial isoprenoid biosynthesis genes during development and osmotic stress responses in Arabidopsis thaliana. BMC Systems Biology 5: 77.

Myśliwa-Kurdziel B, Turek E, Malec P (2011) Protochlorophyllide forms in etiolated seedlings of photoreceptor mutants of Arabidopsis thaliana - is chlorophyll biosynthesis controlled by cooperation between phytochromes and phototropins? Proceedings of the 15th International Congress on Photosynthesis, August 22-27, 2010 Beijing, China (in press).

Myśliwa-Kurdziel B, Strzałka K (2010) Light-induced reduction of protochlorophyllide in angiosperms and chloroplast development. Postepy Biochemii 56: 418-426 (in Polish).

Park H, Kreunen SS, Cuttriss AJ, DellaPenna D, Pogson BJ (2002) Identification of the carotenoid isomerase provides insight into carotenoid biosynthesis, prolamellar body formation, and photomorphogenesis. The Plant Cell 14: 321-332.

Rodriguez-Villalón A, Gas E, Rodriguez-Concepción M (2009) Colors in the dark. A model for the regulation of carotenoid biosynthesis in etioplasts. Plant Signaling and Behaviour 4: 965-967.

Schoefs B, Bertrand M, Lemoine Y (1995) Separation of photosynthetic pigments and their precursors by reversed-phase high-performance liquid chromatography using a photodiode-array detector. J Chromatography 692: 239-245.

Schoefs B (2005) Protochlorophyllide reduction - what is new in 2005? Photosynthetica 43: 329-343.

Schoefs B, Franck F (2003) Protochlorophyllide reduction: mechanisms and evolution. Photochem Photobiol 78: 543-557.

Solymosi K, Schoefs B (2010) Etioplast and etio-chloroplast formation under natural conditions:the dark side of chlorophyll biosynthesis in angiosperms. Photosynth Res 105: 143-166.

Yahubyan G, Minkov I, Sundqvist C (2001) Carotenoid dependence of the protochlorophyllide to chlorophyllide phototransformation in dark-grown wheat seedlings. J Photochem Photobiol 65: 171-176.

Yanovsky, MJ, Casal JJ, Luppi JP (1997) The VLF loci, polymorphic between ecotypes Landsberg erecta and Columbia dissect two branches of phytochrome A signalling pathways that correspond to the very-low fluence and high-irradiance responses of phytochrome. Plant J 12, 659-667.

Von Arnim A, Deng XW (1996) Light control of seedling development. Annu Rev Plant Physiol Plant Mol Biol 47: 215-243.

Welsch R, Beyer P, Hugueney P, Kleinig H, von Lintig J (2000) Regulation and activation of phytoene synthese, a key enzyme in carotenoid biosynthesis, during photomorphogenesis. Planta 211: 846-854. 